\title{
Overcoming Proteasome Inhibitor-Refractory Multiple Myeloma With Elotuzumab, Bortezomib, Nelfinavir, and Dexamethasone
}

\author{
Alexandra Erath ${ }^{\mathrm{a}}$, Dilan A. Patel ${ }^{\mathrm{b}, \mathrm{f}}$, Eric A. Hosack ${ }^{\mathrm{c}}$, James E. Patanella ${ }^{\mathrm{d}}$, \\ Daniel M. Ibach ${ }^{\mathrm{e}}$, Adetola A. Kassim ${ }^{\mathrm{b}}$
}

\begin{abstract}
Multiple myeloma is a common plasma cell malignancy with a median overall survival of fewer than 10 years. Proteasome inhibitors comprise an important part of the treatment regimen for this disease. The present study reports the case of a 57-year-old man who experienced a second relapse of multiple myeloma 6 years after initial treatment with bortezomib, lenalidomide, dexamethasone (VRD) followed by autologous hematopoietic cell transplant. The first relapse had been successfully treated with VRD, but this approach failed to control his second relapse. Given the lack of response to VRD therapy and relapse while on bortezomib maintenance, the patient was deemed proteasome inhibitor-refractory and received a new treatment of elotuzumab, lenalidomide, and dexamethasone. Four and a half cycles were completed before the treatment was stopped due to grade 4 cytopenias. The patient received a novel combination of elotuzumab, bortezomib, nelfinavir, and dexamethasone. After six cycles, the serum M-protein level was improved to $0.6 \mathrm{~g} / \mathrm{dL}$ and the kappa light chains dropped from 3.49 to $1.04 \mathrm{mg} / \mathrm{dL}$. A bone marrow biopsy conducted after five treatment cycles demonstrated $<1 \%$ plasma cells by immunohistochemistry and achievement of minimal residual disease status. Overall, this case study suggests that proteasome inhibitor-refractory multiple myeloma may be successfully re-treated with proteasome inhibitors when co-administered with nelfinavir.
\end{abstract}

Keywords: Myeloma; Nelfinavir; Elotuzumab; Proteasome-inhibitor refractory

Manuscript submitted September 23, 2019, accepted October 22, 2019

${ }^{a}$ Vanderbilt University School of Medicine, Nashville, TN, USA

${ }^{b}$ Department of Hematology and Bone Marrow Transplant, Vanderbilt-Ingram Cancer Center, Vanderbilt University Medical Center, Nashville, TN, USA

'Vanderbilt University, Nashville, TN, USA

dVanderbilt Ingram Cancer Center, 1301 Medical Center Drive \#1710, Nashville, TN 37232, USA

'Tennessee Cancer Specialists, Knoxville, TN, USA

${ }^{\mathrm{f} C}$ Corresponding Author: Dilan A. Patel, Department of Medicine, Division of Hematology/Oncology, Vanderbilt University Medical Center, Nashville, TN 37232, USA. Email: dilan.a.patel.1@vumc.org

doi: https://doi.org/10.14740/wjon1241

\section{Introduction}

Multiple myeloma (MM) is a common hematological malignancy of plasma cells with a median overall survival of $8-10$ years for standard risk patients and 3 years for high risk patients [1]. While recent advances in the field have improved the prognosis for newly diagnosed patients [2], treatment options for relapsed/refractory MM patients are limited. This category includes patients who experienced relapse after a primary remission or who did not achieve remission in initial therapy. Retreatment with prior agents, while an option if the patient had previously responded to the treatment with a remission period of at least 6 months, typically results in a much smaller treatment effect [3]. The proteasome inhibitor (PI) bortezomib has demonstrated efficacy for relapsed myeloma patients refractory to conventional immunomodulatory therapy [4]. However, many patients eventually become refractory to bortezomib, and the prognosis of relapsed PI-refractory myeloma is especially poor $[4,5]$. Patients who become refractory to bortezomib also show a greatly reduced response, in the $20-30 \%$ range, to other next-generation therapies like carfilzomib or pomalidomide, leaving oncologists with few treatment options [6].

A possible new option for patients with PI-refractory myeloma is co-administration of the antiretroviral drug nelfinavir to re-sensitize the myeloma cells to the unfolded protein response (UPR) degradation pathway. A recent phase II study found that treatment of PI-refractory MM patients with nelfinavir in combination with bortezomib and dexamethasone achieved an overall response rate of $65 \%$ [7]. However, there is currently a need for further research into the use of nelfinavir in relapsed/ refractory myeloma patients, especially in conjunction with additional anti-myeloma agents. The present study reports the successful treatment of a 57-year-old MM patient in which PIrefractor MM was overcome by a novel treatment combination of nelfinavir, elotuzumab, bortezomib, and dexamethasone.

\section{Case Report}

A 57-year-old man was admitted on October 27, 2010 for further evaluation of a hemoglobin level of $7 \mathrm{~g} / \mathrm{dL}$. Two days later, the patient was diagnosed with stage II immunoglobulin $\mathrm{G}(\mathrm{IgG}) \lambda$ light chain MM. He received five cycles of induction 


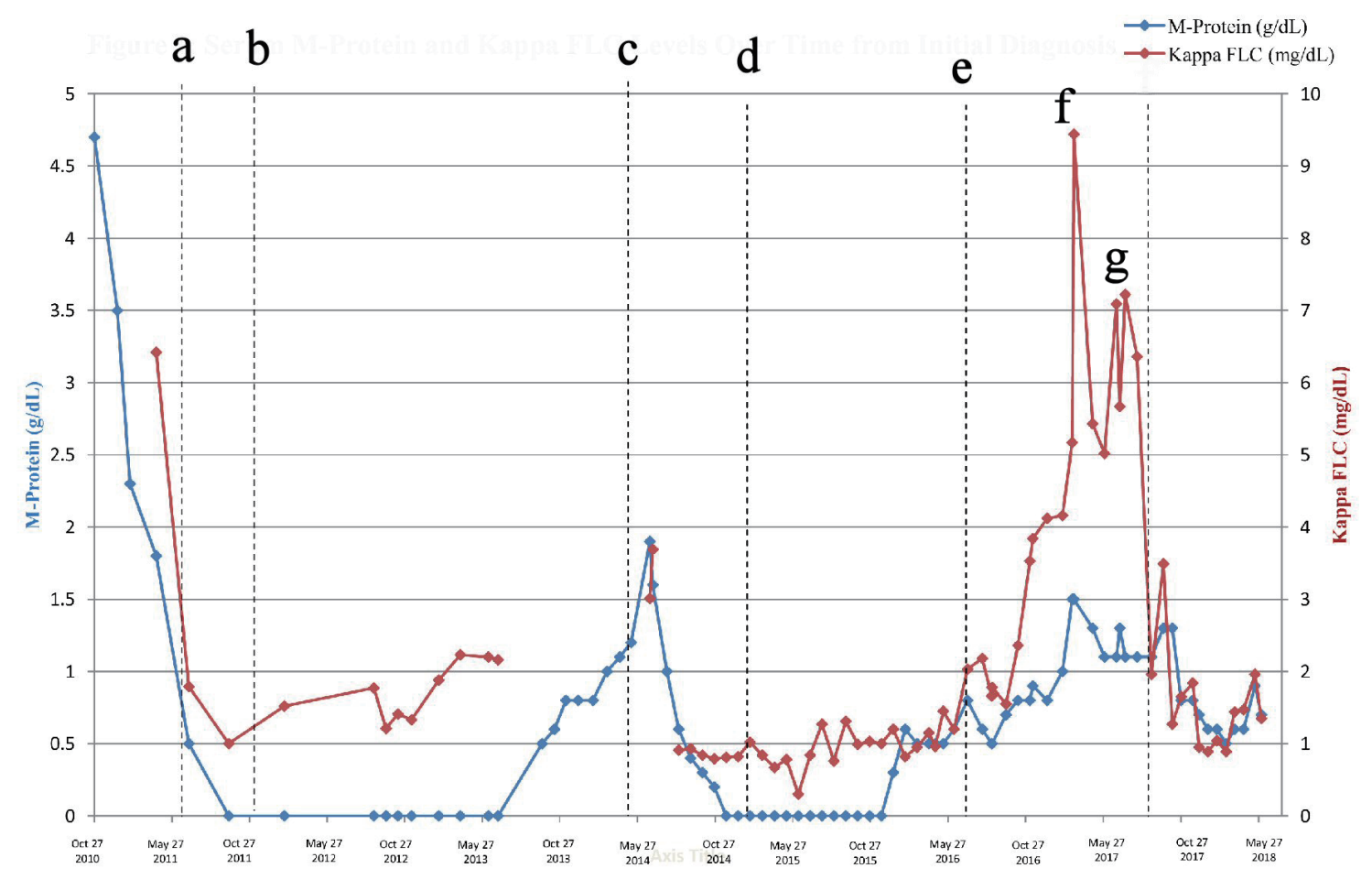

Figure 1. Serum M-protein and kappa FLC levels over time from initial diagnosis. a: Induction therapy: lenalidomide (25 mg); dexamethasone $(40 \mathrm{mg})$; and added bortezomib $\left(1.3 \mathrm{mg} / \mathrm{m}^{2}\right)$ in April 2011. b: Stem cell collection and AHCT, resulting in a VGPR. c: Maintenance therapy with lenalidomide (15 mg, days 1 - 21), 28-day cycles, resulted in remission. d: First relapse: treatment with RVd (lenalidomide $15 \mathrm{mg}$, bortezomib $1.3 \mathrm{mg} / \mathrm{m}^{2}$, dexamethasone $40 \mathrm{mg}$ ). e: Maintenance therapy with bortezomib $1.3 \mathrm{mg} /$ $\mathrm{m}^{2}$, added dexamethasone $(40 \mathrm{mg}$ ) on days of bortezomib dosing in January 2016. f: Second relapse. The following treatments were tried and failed: Rvd, ixazomib monotherapy, ixazomib + lenalidomide, elotuzumab + Rd. g: Treatment with nelfinavir, elotuzumab, bortezomib, and dexamethasone of 21-day cycles started on Aug 8, 2017. FLC: free light chains; AHCT: autologous hematopoietic cell transplant; VGPR: very good partial response; RVd: lenalidomide, bortezomib, dexamethasone; Rd: lenalidomide and dexamethasone.

therapy consisting of 28-day cycles of lenalidomide and dexamethasone and a sixth month with the addition of bortezomib (Supplementary Material 1, www.wjon.org). On July 22, 2011, the patient underwent an autologous hematopoietic cell transplant (AHCT), and achieved a very good partial response. At day +90 , the patient began single agent maintenance therapy with lenalidomide.

In February 2014, the serum M-protein began to rise. Bone marrow biopsy on May 29, 2014 showed 20\% plasma cells by cluster of differentiation (CD)138 staining. In June 2014, the patient began treatment with bortezomib, lenalidomide, and dexamethasone (VRD) (Supplementary Material 1, www. wjon.org). After completing six 28-day cycles, the serum Mprotein level decreased to an undetectable number and a bone marrow biopsy (January 12, 2015) showed $<5 \%$ plasma cells (Fig. 1). Having again achieved remission status, the patient began single agent maintenance therapy with bortezomib. On December 22, 2015, the M-protein rose to $0.3 \mathrm{~g} / \mathrm{dL}$. Starting January 19, 2016, dexamethasone was added to the maintenance treatment schedule.

Over the next 4 months, the serum M-protein level remained stable at approximately $0.5 \mathrm{~g} / \mathrm{dL}$ until a spike to 0.8 $\mathrm{g} / \mathrm{dL}$ in June 2016. After he failed to respond to retreatment with VRD, several other treatment regimens were substituted over the following 6-month period (Supplementary Material 1, www.wjon.org). On August 28, 2016, the patient switched to ixazomib as single agent therapy and completed two cycles, but the serum M-protein continued to increase. He then completed a cycle of lenalidomide and ixazomib with no change in response. By January 2017, another attempt using VRD failed to provide any improvement and the patient's M-protein level had risen to $1.0 \mathrm{~g} / \mathrm{dL}$.

A bone marrow biopsy on February 20, 2017 confirmed disease recurrence with $40 \%$ plasma cells. As the patient had relapsed while on bortezomib maintenance and had failed subsequent treatment with both bortezomib and ixazomib, the patient was deemed PI-refractory, and PIs were excluded from the next treatment choices. After a 6-week break, the patient began treatment with elotuzumab, lenalidomide, and dexamethasone on March 8, 2017. After three cycles, a marginal improvement had been achieved, and the patient received platelet and red blood cell transfusions along with regular filgrastim injections to help bolster his counts during treatment.

Despite these precautions, the patient's counts had dropped precipitously by day 15 of the fifth cycle of treatment, with platelets of $6 \times 10^{3} / \mu \mathrm{L}$, hemoglobin $10.5 \mathrm{~g} / \mathrm{dL}$, and neutrophils $0.28 \times 10^{3} / \mu \mathrm{L}$, prompting the patient's local oncologist to halt treatment due to safety concerns. 
The patient had learned of several promising new studies demonstrating the efficacy of the antiretroviral drug nelfinavir, originally developed for human immunodeficiency virus (HIV) therapy, in combination with bortezomib and dexamethasone in PI-refractory MM patients [6-8]. The patient's local oncologist added elotuzumab, hypothesizing that the addition of a second anti-myeloma drug might provide a more potent therapy and a deeper response. Due to thrombocytopenia, the treatment schedule was adjusted to skip the day 8 dose of bortezomib and the day 9 dose of dexamethasone, and to use a 1.2 $\mathrm{mg} / \mathrm{m}^{2}$ dose of bortezomib instead of a $1.3 \mathrm{mg} / \mathrm{m}^{2}$ dose.

The chemotherapy dosing regimen used for the patient consisted of nelfinavir, bortezomib, elotuzumab, and dexamethasone (Supplementary Material 2, www.wjon.org). The patient began the first cycle on August 8, 2017. Partial laboratory studies during initial treatment cycle are included (Supplementary Material 2, www.wjon.org).

After one cycle, kappa free light chains dropped from 6.36 to $1.96 \mathrm{mg} / \mathrm{dL}$. Due to thrombocytopenia, the second cycle was postponed 2 weeks and shortened to 14 days of treatment, with the patient off all medications for days 8 - 14 (Supplementary Material 2, www.wjon.org). For this second cycle, the patient received elotuzumab (10 $\mathrm{mg} / \mathrm{kg}$ intravenous) on day 1 , bortezomib $\left(1.2 \mathrm{mg} / \mathrm{m}^{2}\right.$ subcutaneous $)$ on day 1 , nelfinavir (2,500 mg orally twice daily) on days $1-7$, dexamethasone ( 8 $\mathrm{mg}$ intravenous and $8 \mathrm{mg}$ orally) on day 1 and dexamethasone (20 $\mathrm{mg}$ orally on day 2). Laboratory results indicated that the 14-day cycle was not effective in controlling the disease.

On September 26, 2017, 21-day cycles of nelfinavir, bortezomib, dexamethasone, and elotuzumab were resumed. He continued to respond well to therapy with decreasing serum M-protein and kappa light chains. Beginning with the fourth cycle, a dose of bortezomib $\left(1.2 \mathrm{mg} / \mathrm{m}^{2}\right.$, subcutaneous) was added on day 8 of the 21-day treatment schedule, as the patient's platelet counts had increased by this point. After five consecutive 21-day cycles, a bone marrow biopsy on January 10,2018 showed that the patient had achieved minimal residual disease negative status by multiparameter flow cytometry.

Biochemical evidence of recurrence was detected based on an increase in serum paraprotein assessed on July 10, 2018 compared to November 13, 2018, with free light chain ratio increasing from 4.16 to 7.61 and M-spike from 0.8 to $0.9 \mathrm{~g} /$ $\mathrm{dL}$, respectively. Treatment was continued with routine monitoring. However, based on labs from February 14, 2019, with free light chain ratio of 15.92 and serum M-spike of $1.5 \mathrm{~g} /$ $\mathrm{dL}$, ixazomib was replaced with lenalidomide with continuation of elotuzumab, nelfinavir, and dexamethasone. The next bone marrow biopsy on August 12, 2019 showed 45\% atypical plasma cells, consistent with involvement by known plasma cell neoplasm. The patient is now being considered for multidrug induction therapy followed by AHCT.

\section{Discussion}

PIs target myeloma cells by inhibiting protein degradation, leading to excessive unfolded protein accumulation and a triggering of the UPR degradation pathway. The UPR is a homeostatic signaling pathway of the endoplasmic reticulum which responds to accumulating proteins by decreasing levels of protein synthesis and increasing transcription of protein chaperones and proteasomes; continued accumulation and endoplasmic reticulum stress eventually lead to UPR-mediated cell-cycle arrest and eventual apoptosis [9]. Fairly unique to myeloma cells is their reliance on a basal level of UPR stimulation due to the large volume of immunoglobulin production, which may predispose triggering of the UPR-apoptotic pathway at relatively lower levels of interference with protein degradation as compared to normal cells [8]. Bortezomib is a selective inhibitor of the $\beta 5$ subunit of the $26 \mathrm{~S}$ proteasome, which exhibits three enzymatically active sites on the $\beta 1, \beta 2$, and $\beta 5$ subunits. HIV protease inhibitors also act on the $26 \mathrm{~S}$ proteasome and similarly aim to promote protein accumulation and induce apoptosis [10]. Nelfinavir has been highlighted as the most promising HIV-1 protease inhibitor for myeloma treatment because it exhibits the highest level of $\beta 5$ inhibition, in addition to being the sole agent to demonstrate effective inhibition of both $\beta 2$ and $\beta 5$ proteasome active sites. $\beta 2$ inhibition has not yet been widely studied as a myeloma therapeutic goal, but bortezomib-resistant myeloma cells have shown increased $\beta 2$ PI activity in comparison to nonresistant cells [11].

Despite the drugs' common $\beta 5$ target, which is associated with the rate-limiting step in proteolysis, myeloma cells refractory to bortezomib have been shown to be susceptible to nelfinavir $[11,12]$. The mechanism of MM resistance to bortezomib is multifaceted, but one proposed mechanism is the upregulation of PI $\beta 2$ subunits. Nelfinavir's action on the $\beta 2$ subunit may contribute to its effectiveness on bortezomib-refractory MM. More importantly, nelfinavir has been shown to increase the expression of UPR-inducing genes inositol-requiring enzyme 1/X-box-binding protein-1 (IRE1/XBP1), resulting in activation of the UPR and res-sensitization in proteasomerefractory cells [6]. Although both bortezomib and nelfinavir molecules bind to the $\beta 5$ active site of the $26 \mathrm{~S}$ proteasome, the co-administration of both agents results in an additive proteasome inhibition and a synergistic effect [11], indicating that nelfinavir is best incorporated into a regimen along with bortezomib. Further research is needed to fully understand its exact molecular actions in conjunction with bortezomib.

\section{Conclusions}

The case reported here demonstrates the remarkable response of one relapsed PI-refractor MM patient with a combination of elotuzumab, bortezomib, nelfinavir, and dexamethasone. The recent use of nelfinavir as a treatment option for PI-refractory $\mathrm{MM}$ is an exciting new discovery in myeloma treatment, though further studies are needed to further explore its usefulness and standardize its treatment regimen prior to application to a larger group of patients.

\section{Supplementary Material}

Suppl. 1. Chemotherapy Dosing Regimen From Diagnosis 
Through Current Novel Regimen (2010 - 2018).

Suppl. 2. Novel Chemotherapy Dosing Regimen of Nelfinavir, Dexamethasone, Bortezomib, and Elotuzumab.

\section{Acknowledgments}

The authors would like to acknowledge the patients and staff of the Vanderbilt University Medical Center and the Division of Hematology and Oncology for this paper.

\section{Financial Disclosure}

The authors do not have any financial disclosures.

\section{Conflict of Interest}

The authors do not have any conflicts of interest to disclose.

\section{Informed Consent}

Informed consent was obtained by the patient prior to writing the case report.

\section{Author Contributions}

AE collected data and wrote the manuscript. DP contributed to writing the manuscript. EH, JP, and DI analyzed data and provided revisions. AK provided the conceptual basis for the treatment plan.

\section{References}

1. Chesi M, Bergsagel PL. Molecular pathogenesis of multiple myeloma: basic and clinical updates. Int J Hematol. 2013;97(3):313-323.

2. Fonseca R, Abouzaid S, Bonafede M, Cai Q, Parikh $\mathrm{K}$, Cosler L, Richardson P. Trends in overall survival and costs of multiple myeloma, 2000-2014. Leukemia.
2017;31(9):1915-1921.

3. Nooka AK, Kastritis E, Dimopoulos MA, Lonial S. Treatment options for relapsed and refractory multiple myeloma. Blood. 2015;125(20):3085-3099.

4. Kane RC, Bross PF, Farrell AT, Pazdur R. Velcade: U.S. FDA approval for the treatment of multiple myeloma progressing on prior therapy. Oncologist. 2003;8(6):508513.

5. Cornell RF, Kassim AA. Evolving paradigms in the treatment of relapsed/refractory multiple myeloma: increased options and increased complexity. Bone Marrow Transplant. 2016;51(4):479-491.

6. Driessen C, Kraus M, Joerger M, Rosing H, Bader J, Hitz F, Berset C, et al. Treatment with the HIV protease inhibitor nelfinavir triggers the unfolded protein response and may overcome proteasome inhibitor resistance of multiple myeloma in combination with bortezomib: a phase I trial (SAKK 65/08). Haematologica. 2016;101(3):346355.

7. Driessen C, Muller R, Novak U, et al. The HIV protease inhibitor Nelfinavir in Combination with Bortezomib and Dexamethasone (NVd) has excellent activity in patients with advanced, proteasome inhibitor-refractory multiple myeloma: a multicenter phase II trial (SAKK 39/13). Blood. 2016;128:487.

8. Bono C, Karlin L, Harel S, Mouly E, Labaume S, Galicier $\mathrm{L}$, Apcher S, et al. The human immunodeficiency virus-1 protease inhibitor nelfinavir impairs proteasome activity and inhibits the proliferation of multiple myeloma cells in vitro and in vivo. Haematologica. 2012;97(7):11011109.

9. Zaal E, Wu W, Jansen G, Zweegman S, Cloos J, Berkers C. Bortezomib resistance in multiple myeloma is associated with increased serine synthesis. Cancer Metab. 2017;5(7):1-12.

10. Yu L, Mohanram V, Simonson OE, Smith CI, Spetz AL, Mohamed AJ. Proteasome inhibitors block HIV-1 replication by affecting both cellular and viral targets. Biochem Biophys Res Commun. 2009;385(1):100-105.

11. Ruckrich T, Kraus M, Gogel J, Beck A, Ovaa H, Verdoes $\mathrm{M}$, Overkleeft HS, et al. Characterization of the ubiquitinproteasome system in bortezomib-adapted cells. Leukemia. 2009;23(6):1098-1105.

12. Lu S, Wang J. The resistance mechanisms of proteasome inhibitor bortezomib. Biomark Res. 2013;1(1):13. 